\title{
$k$-TUPLE TOTAL DOMINATION IN INFLATED GRAPHS
}

\author{
ADEL P. KAZEMI \\ DEPARTMENT OF MATHEMATICS \\ UNIVERSITY OF MOHAGHEGH ARDABILI \\ P. O. BOX 5619911367, ARDABIL, IRAN \\ ADELPKAZEMI@YAHOO.COM
}

\begin{abstract}
The inflated graph $G_{I}$ of a graph $G$ with $n(G)$ vertices is obtained from $G$ by replacing every vertex of degree $d$ of $G$ by a clique, which is isomorph to the complete graph $K_{d}$, and each edge $\left(x_{i}, x_{j}\right)$ of $G$ is replaced by an edge $(u, v)$ in such a way that $u \in X_{i}, v \in X_{j}$, and two different edges of $G$ are replaced by non-adjacent edges of $G_{I}$. For integer $k \geq 1$, the $k$-tuple total domination number $\gamma_{\times k, t}(G)$ of $G$ is the minimum cardinality of a $k$-tuple total dominating set of $G$, which is a set of vertices in $G$ such that every vertex of $G$ is adjacent to at least $k$ vertices in it. For existing this number, must the minimum degree of $G$ is at least $k$. Here, we study the $k$-tuple total domination number in inflated graphs when $k \geq 2$. First we prove that $n(G) k \leq \gamma_{\times k, t}\left(G_{I}\right) \leq n(G)(k+1)-1$, and then we characterize graphs $G$ that the $k$-tuple total domination number number of $G_{I}$ is $n(G) k$ or $n(G) k+1$. Then we find bounds for this number in the inflated graph $G_{I}$, when $G$ has a cut-edge $e$ or cut-vertex $v$, in terms on the $k$-tuple total domination number of the inflated graphs of the components of $G-e$ or $v$-components of $G-v$, respectively. Finally, we calculate this number in the inflated graphs that have obtained by some of the known graphs.
\end{abstract}

Keywords : $k$-tuple total domination number, inflated graph 2000 Mathematics subject classification : 05C69

\section{INTRODUCTION}

All graphs considered here are finite, undirected, and simple. For standard graph theory terminology not given here we refer to [2]. Let $G=(V, E)$ be a graph with vertex set $V$ of $\operatorname{order} n(G)$ and edge set $E$ of size $m(G)$. The open neighborhood of a vertex $v \in V$ is $N_{G}(v)=\{u \in V \mid u v \in E\}$ and its closed neighborhood is $N_{G}[v]=N_{G}(v) \cup\{v\}$. The degree of a vertex $v$ is also $\operatorname{deg}_{G}(v)=\left|N_{G}(v)\right|$. The minimum and maximum degree of $G$ are respectively denoted by $\delta=\delta(G)$ and $\Delta=\Delta(G)$. We say that a graph is connected if there exist a path between every two vertices of the graph, and otherwise is called disconnected. In a connected graph $G$, a vertex (resp. edge) $v$ is called a cut-vertex or (resp. cut-edge) if $G-v$ is disconnected. Every maximal connected subgraph of $G-v$ is called a (connectedness) component of it. Let $v$ be a cut-vertex of a graph $G$ and $S$ be the vertex set of a component of $G-v$. The induced subgraph by $S \cup\{v\}$ of $G$ we call a $v$-component of $G$.

An edge subset $M$ in $G$ is called a matching in $G$ if any two edges of $M$ has no vertex in common. If $e=v w \in M$, then we say either $M$ saturate two vertices $v$ and $w$ or $v$ and $w$ are $M$-saturated (by $e$ ). A matching $\mathrm{M}$ is a perfect matching if all vertices of $G$ are $M$-saturated. Also a matching $M$ is a maximum matching if there is no other matching $M^{\prime}$ with $\left|M^{\prime}\right|>|M|$. In a graph $G$ the number of edges in a maximum matching is denoted by $\alpha^{\prime}(G)$.

Domination in graphs is now well studied in graph theory and the literature on this subject has been surveyed and detailed in the two books by Haynes, Hedetniemi, and Slater 2, 3. A set $S \subseteq V$ is a total dominating set if each vertex in $V$ is adjacent to at least one vertex of $S$, while the minimum cardinality of a total dominating set is the total domination number $\gamma_{t}(G)$ of $G$.

In 4 Henning and Kazemi generalized this definition to the $k$-tuple total domination number as follows: a subset $S$ of $V$ is a $k$-tuple total dominating set of $G$, abbreviated kTDS, if for every vertex $v \in V,|N(v) \cap S| \geq k$; that is, $S$ is a kTDS if every vertex has at least $k$ neighbors in $S$. The $k$-tuple 
total domination number $\gamma_{\times k, t}(G)$ of $G$ is the minimum cardinality of a kTDS of $G$. We remark that $\gamma_{t}(G)=\gamma_{\times 1, t}(G)$. For a graph to have a $k$-tuple total dominating set, its minimum degree is at least $k$. Since every $(\mathrm{k}+1)$ TDS is also a kTDS, we note that $\gamma_{\times k, t}(G) \leq \gamma_{\times(k+1), t}(G)$ for all graphs with minimum degree at least $k+1$. A kTDS of cardinality $\gamma_{\times k, t}(G)$ we call a $\gamma_{\times k, t}(G)$-set. When $k=2$, a $k$-tuple total dominating set is called a double total dominating set, abbreviated DTDS, and the $k$-tuple total domination number is called the double total domination number. The redundancy involved in $k$-tuple total domination makes it useful in many applications.

For the notation for inflated graphs, we follow that of [7]. The infation or infated graph $G_{I}$ of the graph $G$ without isolated vertices is obtained as follows: each vertex $x_{i}$ of degree $d\left(x_{i}\right)$ of $G$ is replaced by a clique $X_{i} \cong K_{d\left(x_{i}\right)}$ (that is, $X_{i}$ is isomorph to the complete graph $K_{d\left(x_{i}\right)}$ ) and each edge $\left(x_{i}, x_{j}\right)$ of $G$ is replaced by an edge $(u, v)$ in such a way that $u \in X_{i}, v \in X_{j}$, and two different edges of $G$ are replaced by non-adjacent edges of $G_{I}$. An obvious consequence of the definition is that $n\left(G_{I}\right)=\sum_{x_{i} \in V(G)} d_{G}\left(x_{i}\right)=2 m(G), \delta\left(G_{I}\right)=\delta(G)$ and $\Delta\left(G_{I}\right)=\Delta(G)$. There are two different kinds of edges in $G_{I}$. The edges of the clique $X_{i}$ are colored red and the $X_{i}$ 's are called the red cliques (a red clique $X_{i}$ is reduced to a point if $x_{i}$ is a pendant vertex of $G$ ). The other ones, which correspond to the edges of $G$, are colored blue and they form a perfect matching of $G_{I}$. Every vertex of $G_{I}$ belongs to exactly one red clique and one blue edge. Two adjacent vertices of $G_{I}$ are said to red-adjacent if they belong to a same red clique, blue-adjacent otherwise. In general, we adopt the following notation: if $x_{i}$ and $x_{j}$ are two adjacent vertices of $G$, the end vertices of the blue edge of $G_{I}$ replacing the edge $\left(x_{i}, x_{j}\right)$ of $G$ are called $x_{i} x_{j}$ in $X_{i}$ and $x_{j} x_{i}$ in $X_{j}$, and this blue edge is $\left(x_{i} x_{j}, x_{j} x_{i}\right)$. Clearly an inflation is claw-free. More precisely, $G_{I}$ is the line-graph $L(S(G))$ where the subdivision $S(G)$ of $G$ is obtained by replacing each edge of $G$ by a path of length 2 . The study of various domination parameters in inflated graphs was originated by Dunbar and Haynes in [6]. Results related to the domination parameters in inflated graphs can be found in [7, 8, 9,

Henning and Kazemi in [5] discussed on total domination number in inflated graphs which is the same $k$-tuple total domination number when $k=1$. Here we continue the studying of the $k$ tuple total domination number in inflated graphs when $k \geq 2$. This paper is organized as follows. In section 2, we prove that if $k \geq 2$ is an integer and $G$ is a graph of order $n$ with $\delta \geq k$, then $n k \leq \gamma_{\times k, t}\left(G_{I}\right) \leq n(k+1)-1$, and then we characterize graphs $G$ that $\gamma_{\times k, t}\left(G_{I}\right)$ is $n k$ or $n k+1$. In section 3 , we find upper and lower bounds for the $k$-tuple total domination number of the inflation of a graph $G$, which contains a cut-edge $e$, in terms on the $k$-tuple total domination number of the inflation of the components of $G-e$. Also in a similar manner, we find upper and lower bounds for the $k$-tuple total domination number of the inflation of a graph $G$, which contains a cut-vertex $v$, in terms on the $k$-tuple total domination number of the inflation of the $v$-components of $G-v$. Also we find the $k$-tuple total domination number of the inflation of the complete graphs. Finally, in section 4 , we calculate the $k$-tuple total domination number in the inflation of the known graphs: the generalized Petersen graphs, Harary graphs and complete bipartite graphs. Also we give an upper bound for this number in the inflation of the complete multipartite graphs.

\section{GENERAL BBOUNDS}

First we give two general upper and lower bounds for the $k$-tuple total domination number of inflated graphs, where $\delta \geq k \geq 2$.

Theorem 1. Let $k \geq 2$ be an integer, and let $G$ be a graph of order $n$ with $\delta \geq k$. Then

$$
n k \leq \gamma_{\times k, t}\left(G_{I}\right) \leq n(k+1)-1 .
$$

Proof. Let $V(G)=\left\{x_{i} \mid 1 \leq i \leq n\right\}$ and let $S$ be an arbitrary kTDS of $G_{I}$. Since every vertex of the red clique $X_{i}$ is adjacent to only one vertex of another red clique, then $\left|S \cap X_{i}\right| \geq\left|N_{X_{i}}[v]\right| \geq k$, for each vertex $v \in S \cap X_{i}$ and hence $\gamma_{\times k, t}\left(G_{I}\right) \geq n k$.

Now we prove $\gamma_{\times k, t}\left(G_{I}\right) \leq n(k+1)-1$. Set $S_{1}=\left\{x_{1} x_{j} \mid 2 \leq j \leq k+1\right\}$ as a subset of $X_{1}$. For each $2 \leq j \leq n$, let $S_{j}$ be a $(k+1)$-subset of $X_{j}$ such that $x_{j} x_{1} \in S_{j}$, for each $2 \leq j \leq k+1$. Since $S_{1} \cup S_{2} \cup \ldots \cup S_{n}$ is a kTDS of $G_{I}$ with cardinal $n(k+1)-1$, then $\gamma_{\times k, t}\left(G_{I}\right) \leq n(k+1)-1$.

We recall the next proposition from 4 . 
Proposition A. (Kazemi, Henning 4 2010) Let $G$ be a graph with minimum degree at least $k$. If $k \geq 2$ is an integer, then

$$
\gamma_{\times k, t}(G) \geq\left\lceil\frac{k n}{\Delta(G)}\right\rceil .
$$

By Proposition $\mathrm{A}$ and Theorem 1 we have the next result.

Corollary 2. If $G$ is a graph of order $n$ and size $m$ with $\delta(G) \geq k \geq 2$, then

$$
\gamma_{\times k, t}\left(G_{I}\right) \geq \max \{n k,\lceil 2 k m / \Delta(G)\rceil\} .
$$

Let $k=\delta(G)$. Then, since every red clique of cardinal $k$ is subset of every kTDS of $G_{I}$, Theorem 1 can be improved in such a way.

Corollary 3. Let $G$ be a graph of order $n$ with $\delta \geq 2$. If $\ell$ is the number of vertices in $G$ of degree $\delta$, then $n \delta \leq \gamma_{\times \delta, t}\left(G_{I}\right) \leq n(\delta+1)-\ell$.

Now, we characterize graphs $G$ of order $n$ that the $k$-tuple total domination number of their inflation is $n k$ or $n k+1$. First we give the next two new definitions.

Two new definitions:

We know that a graph $G$ is a Hamiltonian graph if it has a Hamiltonian cycle, that is, a cycle that contains all vertices of the graph. We extend this definition in such a way: a graph $G$ is a Hamiltonian-like decomposable graph if there are disjoint Hamiltonian subgraphs $G_{1}, G_{2}, \ldots, G_{t}$ of $G$ such that $V(G)=V\left(G_{1}\right) \cup V\left(G_{2}\right) \cup \ldots \cup V\left(G_{t}\right)$. A such partition we call a Hamiltonian-like decomposition of $G$ and simply we write $G=H L D\left(G_{1}, G_{2}, \ldots, G_{t}\right)$. In generally, for each integer $k \geq 1$, we say that a graph $G$ is a $k$-Hamiltonian-like decomposable graph, briefly kHLD-graph, if it has $k$ Hamiltonian-like decomposition $G=H L D\left(G_{1}^{(i)}, G_{2}^{(i)}, \ldots, G_{t_{i}}^{(i)}\right)$ of Hamiltonian subgraphs (where $1 \leq i \leq k$ ) such that for every two distinct Hamiltonian subgraphs $G_{s_{i}}^{(i)}$ and $G_{s_{j}}^{(j)}$, their Hamiltonian cycles $C_{s_{i}}^{(i)}$ and $C_{s_{j}}^{(j)}$ are disjoint. We note that 1-Hamiltonian-like decomposable graph is the same Hamiltonian-like decomposable graph.

A $k$-Hamiltonian-like decomposable graph $G$, we call kHLPM-graph or kHLMM-graph if $G$ has respectively a perfect or maximum matching $M$ with cardinal $\lfloor n / 2\rfloor$ such that for each partition $G=H L D\left(G_{1}^{(i)}, G_{2}^{(i)}, \ldots, G_{t_{i}}^{(i)}\right)$ of Hamiltonian subgraphs (where $1 \leq i \leq k$ ), $M$ satisfies in the following condition:

$$
M \cap E\left(C_{\ell_{i}}^{(i)}\right)=\emptyset, \text { for each } 1 \leq \ell_{i} \leq t_{i},
$$

where $C_{\ell_{i}}^{(i)}$ is the Hamiltonian cycle of $G_{\ell_{i}}^{(i)}$.

The next two theorems characterize graphs $G$ with $\gamma_{\times k, t}\left(G_{I}\right)=n k$.

Theorem 4. Let $G$ be a graph of order $n$ and let $1 \leq 2 k \leq \delta$. Then $\gamma_{\times(2 k), t}\left(G_{I}\right)=2 k n$ if and only if $G$ is a kHLD-graph.

Proof. Let $V(G)=\left\{x_{i} \mid 1 \leq i \leq n\right\}$. For each $1 \leq i \leq k$ and some $t_{i} \geq 1$, let $G=H L D\left(G_{1}^{(i)}, G_{2}^{(i)}, \ldots, G_{t_{i}}^{(i)}\right)$ be a Hamiltonian-like decomposition of $G$. For each $1 \leq i \leq k$ and each $1 \leq \ell_{i} \leq t_{i}$, let $C_{\ell_{i}}^{(i)}: x_{1}^{(i)} x_{2}^{(i)} \ldots x_{c_{i, \ell_{i}}}^{(i)}$ be the Hamiltonian cycle of $G_{\ell_{i}}^{(i)}$. Set

$$
S_{i, \ell_{i}}=\left\{x_{m}^{(i)} x_{m-1}^{(i)}, x_{m}^{(i)} x_{m+1}^{(i)} \mid 1 \leq m \leq c_{i, \ell_{i}}\right\} .
$$

Then every $S^{(i)}=S_{i, 1} \cup S_{i, 2} \cup \ldots \cup S_{i, t_{i}}$ is a DTDS of $G_{I}$ with cardinal $2 n$. Since $G$ is $k$-Hamiltonianlike decomposable, then every two distinct $S^{(i)}$ and $S^{(\ell)}$ are disjoint and hence $S^{(1)} \cup S^{(2)} \cup \ldots \cup S^{(k)}$ is a $2 \mathrm{kTDS}$ of $G_{I}$ with cardinal $2 k n$. Thus $\gamma_{\times(2 k), t}\left(G_{I}\right) \leq 2 k n$ and Theorem 1 follows $\gamma_{\times(2 k), t}\left(G_{I}\right)=2 k n$.

Conversely, let $\gamma_{\times(2 k), t}\left(G_{I}\right)=2 k n$ and let $S$ be a $\gamma_{\times(2 k), t}\left(G_{I}\right)$-set. Since for each $1 \leq i \leq n$, $\left|S \cap X_{i}\right|=2 k$, then we may partition every $S \cap X_{i}$ to $k 2$-subsets $D_{j}^{(i)}$, where $1 \leq j \leq k$, such that $D_{j}^{(1)} \cup D_{j}^{(2)} \cup \ldots \cup D_{j}^{(n)}$ is a union of some disjoint cycles. Without loss of generality, we may assume that $D_{j}^{(1)} \cup D_{j}^{(2)} \cup \ldots \cup D_{j}^{(n)}$ is the cycle

$$
C_{j}: x_{1} x_{n}, x_{1} x_{2} ; x_{2} x_{1}, x_{2} x_{3} ; x_{3} x_{2}, x_{3} x_{4} ; \ldots ; x_{n} x_{n-1}, x_{n} x_{1} .
$$


Then $G$ has the corresponding cycle $C_{j}^{\prime}: x_{1} x_{2} x_{3} x_{4} \ldots x_{n}$. Thus for every partition $D_{j}^{(1)} \cup D_{j}^{(2)} \cup \ldots \cup D_{j}^{(n)}$ there is a corresponding partition $G=H L D\left(G_{1}^{(i)}, G_{2}^{(i)}, \ldots, G_{t_{i}}^{(i)}\right)$ of Hamiltonian subgraphs $G_{1}^{(i)}, G_{2}^{(i)}$, $\ldots$ and $G_{t_{i}}^{(i)}$, and so $G$ is a kHLD-graph.

Theorem 5. Let $G$ be a graph of order $n$ and let $1 \leq 2 k+1 \leq \delta$. Then $\gamma_{\times(2 k+1), t}\left(G_{I}\right)=(2 k+1) n$ if and only if $G$ is a kHLPM-graph.

Proof. Let $V(G)=\left\{x_{i} \mid 1 \leq i \leq n\right\}$. Let $G$ be a kHLPM-graph. We follow exactly the notation and terminology introduced in the first and second paragraphs of the proof of Theorem 4 . Then similarly $S^{(1)} \cup S^{(2)} \cup \ldots \cup S^{(k)}$ is a $2 \mathrm{kTDS}$ of $G_{I}$ with cardinal $2 k n$. Set $M_{I}=\left\{\left(x_{i} x_{j}, x_{j} x_{i}\right) \mid x_{i} x_{j} \in M\right\}$. Since for every partition $G=H L D\left(G_{1}^{(i)}, G_{2}^{(i)}, \ldots, G_{t_{i}}^{(i)}\right)$ of Hamiltonian subgraphs, $M$ satisfies in the condition (1), then $V\left(M_{I}\right) \cap\left(S^{(1)} \cup S^{(2)} \cup \ldots \cup S^{(k)}\right)=\emptyset$. One can verify that $V\left(M_{I}\right) \cup S^{(1)} \cup S^{(2)} \cup$ $\ldots \cup S^{(k)}$ is a $(2 \mathrm{k}+1)$ TDS of $G_{I}$ with cardinal $(2 k+1) n$. Thus $\gamma_{\times(2 k+1), t}\left(G_{I}\right) \leq(2 k+1) n$ and Theorem 1 follows $\gamma_{\times(2 k+1), t}\left(G_{I}\right)=(2 k+1) n$.

Conversely, let $\gamma_{\times(2 k+1), t}\left(G_{I}\right)=(2 k+1) n$ and let $S$ be a $\gamma_{\times(2 k+1), t}\left(G_{I}\right)$-set. Since for each $1 \leq i \leq n,\left|S \cap X_{i}\right|=2 k+1$, then, similar to the proof of Theorem 1 we may partition every $S \cap X_{i}$ to $k$ 2-subsets $D_{j}^{(i)}$, where $1 \leq j \leq k$, such that $D_{j}^{(1)} \cup D_{j}^{(2)} \cup \ldots \cup D_{j}^{(n)}$ is a union of some disjoint cycles and there is a corresponding partition $G=H L D\left(G_{1}^{(i)}, G_{2}^{(i)}, \ldots, G_{t_{i}}^{(i)}\right)$ of Hamiltonian subgraphs for it, and also $\cup_{1 \leq i \leq n}\left(S-\left(\cup_{1 \leq j \leq k} D_{j}^{(i)}\right)\right)$ makes a blue matching $M_{I}$ in $G_{I}$ of size $\lfloor n / 2\rfloor$. It can be easily verified that $M=\left\{x_{i} x_{j} \mid\left(x_{i} x_{j}, x_{j} x_{i}\right) \in M_{I}\right\}$ is a perfect matching in $G$ that satisfies in the condition (11), and so $G$ is a kHLPM-graph.

Theorems 14 and 5 follow the next result.

Theorem 6. Let $G$ be a graph of order $n$, and let $1 \leq k \leq \delta$. Then

$$
n k+1 \leq \gamma_{\times k, t}\left(G_{I}\right) \leq n(k+1)-1
$$

if and only if either $k$ and $n$ are both odd or if $k$ is even or odd, then respectively $G$ is not a $k H L D$ or kHLPM-graph.

By closer look at the proofs of Theorems 4 and 5 we have the following observation.

Observation 7. Let $k$ be an integer and let $G$ be a graph of order $n$ with $\gamma_{\times k, t}(G)=n k$. Then for every $\gamma_{\times k, t}\left(G_{I}\right)$-set $S$, the induced subgraph $G_{I}[S]$ of $S$ in $G_{I}$ contains a union of disjoint Hamiltonian cycles (of some of the its subgraphs) and probably a perfect matching. Therefore, if we reduce the number of vertices of $S$ in a red clique of $G_{I}$ to less than $k$ vertices, then there is another unique red clique $X$ of $G_{I}$ and an unique vertex $w$ of $X \cap S$ such that $w$ is not $k$-tuple totally dominated by $S$.

The next theorem states an equivalent condition for $\gamma_{\times k, t}\left(G_{I}\right)=n k+1$, when $k$ and $n$ are both odd.

Theorem 8. Let $G$ be a graph of odd order $n$ and let $1 \leq 2 k+1 \leq \delta$. Then $\gamma_{\times(2 k+1), t}\left(G_{I}\right)=$ $(2 k+1) n+1$ if and only if $G$ is a kHLMM-graph.

Proof. Let $V(G)=\left\{x_{i} \mid 1 \leq i \leq n\right\}$. Let $G$ be a kHLMM-graph. Without loss of generality, we may assume that $M$ does not saturate $x_{n}$. For each $1 \leq i \leq k$ and $t_{i} \geq 1$, let $G=H L D\left(G_{1}^{(i)}, G_{2}^{(i)}, \ldots, G_{t_{i}}^{(i)}\right)$ be a Hamiltonian-like decomposition. For each $1 \leq \ell_{i} \leq t_{i}$, let $C_{\ell_{i}}^{(i)}: x_{1}^{(i)} x_{2}^{(i)} \ldots x_{c_{i, \ell_{i}}}^{(i)}$ be a Hamiltonian cycle for $G_{\ell_{i}}^{(i)}$. Set

$$
S_{i, \ell_{i}}=\left\{x_{m}^{(i)} x_{m-1}^{(i)}, x_{m}^{(i)} x_{m+1}^{(i)} \mid 1 \leq m \leq c_{i, \ell_{i}}\right\} .
$$

Then every $S^{(i)}=S_{i, 1} \cup S_{i, 2} \cup \ldots \cup S_{i, t_{i}}$ is a DTDS of $G_{I}$ with cardinal $2 n$. Since $G$ is $k$-Hamiltonianlike decomposable, then every two distinct $S^{(i)}$ and $S^{(\ell)}$ are disjoint and hence $S^{(1)} \cup S^{(2)} \cup \ldots \cup S^{(k)}$ is a $2 \mathrm{kTDS}$ of $G_{I}$ with cardinal $2 k n$. Set $M_{I}=\left\{\left(x_{i} x_{j}, x_{j} x_{i}\right) \mid x_{i} x_{j} \in M\right\}$. Since for each partition $G=H L D\left(G_{1}^{(i)}, G_{2}^{(i)}, \ldots, G_{t_{i}}^{(i)}\right)$ of Hamiltonian subgraphs, $M$ satisfies in the condition (1), then $V\left(M_{I}\right) \cap\left(S^{(1)} \cup S^{(2)} \cup \ldots \cup S^{(k)}\right)=\emptyset$. One can verify that for every two arbitrary vertices $\alpha, \beta \in$ 
$X_{n}-\left(S^{(1)} \cup S^{(2)} \cup \ldots \cup S^{(k)}\right)$, the set $V\left(M_{I}\right) \cup S^{(1)} \cup S^{(2)} \cup \ldots \cup S^{(k)} \cup\{\alpha, \beta\}$ is a $(2 k+1)$ TDS of $G_{I}$ with cardinal $(2 k+1) n+1$. Thus $\gamma_{\times(2 k+1), t}\left(G_{I}\right) \leq(2 k+1) n+1$ and Theorem 6 follows $\gamma_{\times(2 k+1), t}\left(G_{I}\right)=(2 k+1) n+1$.

Conversely, let $\gamma_{\times(2 k+1), t}\left(G_{I}\right)=(2 k+1) n+1$ and let $S$ be a $\gamma_{\times(2 k+1), t}\left(G_{I}\right)$-set. Without loss of generality, we may assume that for each $1 \leq i \leq n-1,\left|S \cap X_{i}\right|=2 k+1$ and $\left|S \cap X_{n}\right|=2 k+2$. Similar to the proofs of the previous theorems, we may partition every $S \cap X_{i}$ to $k 2$-subsets $D_{j}^{(i)}$, where $1 \leq j \leq k$, such that $D_{j}^{(1)} \cup D_{j}^{(2)} \cup \ldots \cup D_{j}^{(n)}$ is a union of some disjoint cycles and there is a corresponding partition $G=H L D\left(G_{1}^{(i)}, G_{2}^{(i)}, \ldots, G_{t_{i}}^{(i)}\right)$ of Hamiltonian subgraphs for it, and also $\cup_{1 \leq i \leq n-1}\left(S-\left(\cup_{1 \leq j \leq k} D_{j}^{(i)}\right)\right)$ makes a blue matching $M_{I}$ in $G_{I}$ of size $\lfloor n / 2\rfloor$. It can be easily verified that $M=\left\{x_{i} x_{j} \mid\left(x_{i} x_{j}, x_{j} x_{i}\right) \in M_{I}\right\}$ is a maximum matching in $G$ of size $\lfloor n / 2\rfloor$ such that does not saturate $x_{n}$ and for every partition $G=H L D\left(G_{1}^{(i)}, G_{2}^{(i)}, \ldots, G_{t_{i}}^{(i)}\right)$ of Hamiltonian subgraphs it satisfies in the condition (10), and so $G$ is a kHLMM-graph.

\section{3. $k$-TUPLE TOTAL DOMINATION NUMBER IN THE INFLATION OF A CONNECTED GRAPH WHICH HAS A CUT-EDGE OR CUT-VERTEX}

In the next theorem we give upper and lower bounds for the $k$-tuple total domination number of the inflation of a graph $F$ which contains a cut-edge $e$, in terms on the $k$-tuple total domination numbers of the inflation of the components of $F-e$.

Theorem 9. Let $F$ be a graph with a cut-edge e such that $G$ and $H$ are the components of $F-e$. If $2 \leq k \leq \min \{\delta(G), \delta(H)\}$, then

$$
\gamma_{\times k, t}\left(G_{I}\right)+\gamma_{\times k, t}\left(H_{I}\right)-k \leq \gamma_{\times k, t}\left(F_{I}\right) \leq \gamma_{\times k, t}\left(G_{I}\right)+\gamma_{\times k, t}\left(H_{I}\right) .
$$

Proof. Let $V(G)=\left\{x_{i} \mid 1 \leq i \leq n\right\}, V(H)=\left\{y_{i} \mid 1 \leq i \leq m\right\}$. Without loss of generality, we may assume that $e=x_{1} y_{1}$. Then $V\left(F_{I}\right)=V\left(G_{I}\right) \cup V\left(H_{I}\right) \cup\left\{x_{1} y_{1}, y_{1} x_{1}\right\}$ and

$$
\begin{aligned}
E\left(F_{I}\right)= & E\left(G_{I}\right) \cup E\left(H_{I}\right) \cup\left\{\left(x_{1} x_{j}, x_{1} y_{1}\right) \mid x_{1} x_{j} \in X_{1}\right\} \\
\cup & \left\{\left(y_{1} y_{j}, y_{1} x_{1}\right) \mid y_{1} y_{j} \in Y_{1}\right\} \cup\left\{\left(x_{1} y_{1}, y_{1} x_{1}\right)\right\} .
\end{aligned}
$$

Also let $X_{1}^{\prime}=X_{1} \cup\left\{x_{1} y_{1}\right\}$ and $Y_{1}^{\prime}=Y_{1} \cup\left\{y_{1} x_{1}\right\}$. Let $S_{G}$ and $S_{H}$ be respectively $\gamma_{\times k, t}\left(G_{I}\right)$-set and $\gamma_{\times k, t}\left(H_{I}\right)$-set. Since $S_{G} \cup S_{H}$ is a kTDS of $F_{I}$ with cardinal $\gamma_{\times k, t}\left(G_{I}\right)+\gamma_{\times k, t}\left(H_{I}\right)$, then $\gamma_{\times k, t}\left(F_{I}\right) \leq \gamma_{\times k, t}\left(G_{I}\right)+\gamma_{\times k, t}\left(H_{I}\right)$.

Let now $S_{F}$ be a $\gamma_{\times k, t}\left(F_{I}\right)$-set. If $S_{F} \cap\left\{x_{1} y_{1}, y_{1} x_{1}\right\}=\emptyset$, then $S_{F} \cap V\left(G_{I}\right)$ and $S_{F} \cap V\left(H_{I}\right)$ are respectively $k$-tuple total dominating sets of $G_{I}$ and $H_{I}$ and hence

$$
\begin{aligned}
\gamma_{\times k, t}\left(G_{I}\right)+\gamma_{\times k, t}\left(H_{I}\right) & \leq\left|S_{F} \cap V\left(G_{I}\right)\right|+\left|S_{F} \cap V\left(H_{I}\right)\right| \\
& =\left|S_{F}\right| \\
& =\gamma_{\times k, t}\left(F_{I}\right) .
\end{aligned}
$$

Therefore, we assume that $S_{F} \cap\left\{x_{1} y_{1}, y_{1} x_{1}\right\} \neq \emptyset$, and in the next two cases we will complete our proof.

Case 1. $\left|S_{F} \cap\left\{x_{1} y_{1}, y_{1} x_{1}\right\}\right|=1$.

Let $S_{F} \cap\left\{x_{1} y_{1}, y_{1} x_{1}\right\}=\left\{x_{1} y_{1}\right\}$. Then $S_{F} \cap V\left(H_{I}\right)$ is a kTDS of $H_{I}$ and $\left|S_{F} \cap X_{1}\right| \geq k$. Since $k \geq 2$ and each clique of every inflated graph contains at least $k$ vertices of every kTDS and $\left|S_{F} \cap X_{1}\right|>k$ follows $\left|S_{F} \cap Y_{1}^{\prime}\right|=k-1$, then $\left|S_{F} \cap X_{1}\right|=k$. If $\operatorname{deg}_{G}\left(x_{1}\right)=k$, then $\left(S_{F} \cap V\left(G_{I}\right)\right) \cup\left\{x_{i} x_{1} \mid x_{1} x_{i} \in X_{1}\right\}$ is a kTDS of $G_{I}$ with cardinal at most $\left|S_{F} \cap V\left(G_{I}\right)\right|+k$ and hence

$$
\begin{aligned}
\gamma_{\times k, t}\left(G_{I}\right)+\gamma_{\times k, t}\left(H_{I}\right) & \leq\left|S_{F} \cap V\left(G_{I}\right)\right|+k+\left|S_{F} \cap V\left(H_{I}\right)\right| \\
& =\gamma_{\times k, t}\left(F_{I}\right)+k-1 .
\end{aligned}
$$

Otherwise, for every $x_{1} x_{j} \in X_{1}-S_{F},\left(S_{F} \cap V\left(G_{I}\right)\right) \cup\left\{x_{1} x_{j}\right\}$ is a kTDS of $G_{I}$ and hence

$$
\begin{aligned}
\gamma_{\times k, t}\left(G_{I}\right)+\gamma_{\times k, t}\left(H_{I}\right) & \leq\left|\left(S_{F} \cap V\left(G_{I}\right)\right) \cup\left\{x_{1} x_{j}\right\}\right|+\left|S_{F} \cap V\left(H_{I}\right)\right| \\
& =\gamma_{\times k, t}\left(F_{I}\right) .
\end{aligned}
$$

Case 2. $\left|S_{F} \cap\left\{x_{1} y_{1}, y_{1} x_{1}\right\}\right|=2$. 
Since $\left|S_{F} \cap X_{1}^{\prime}\right| \geq k,\left|S_{F} \cap Y_{1}^{\prime}\right| \geq k$ and $\left\{x_{1} y_{1}, y_{1} x_{1}\right\} \subseteq S_{F}$, then $\left|S_{F} \cap X_{1}\right|=k-1$ or $\left|S_{F} \cap Y_{1}\right|=k-1$. Let $\left|S_{F} \cap X_{1}\right| \geq\left|S_{F} \cap Y_{1}\right|=k-1$. If $\operatorname{deg}_{H}\left(y_{1}\right)=k$, then there exists $y_{1} y_{j} \in Y_{1}-S_{F}$ such that $\left(S_{F} \cap V\left(H_{I}\right)\right) \cup\left\{y_{1} y_{j}, y_{j} y_{1}\right\}$ is a kTDS of $H_{I}$. If $\operatorname{deg}_{H}\left(y_{1}\right) \geq k+1$, then there are two disjoint vertices $y_{1} y_{j}, y_{1} y_{i} \in Y_{1}-S_{F}$ such that $\left(S_{F} \cap V\left(H_{I}\right)\right) \cup\left\{y_{1} y_{j}, y_{1} y_{i}\right\}$ is a kTDS of $H_{I}$.

Now we give a $k$-tuple total dominating set for $G_{I}$ in all possible cases. If $\left|S_{F} \cap X_{1}^{\prime}\right| \geq k+1$, then $S_{F} \cap V\left(G_{I}\right)$ is a kTDS of $G_{I}$. Let $\left|S_{F} \cap X_{1}\right|=k$ and let $\operatorname{deg}_{G}\left(x_{1}\right)=k$. Then $\left(S_{F} \cap V\left(G_{I}\right)\right) \cup\left\{x_{i} x_{1} \mid\right.$ $\left.x_{1} x_{i} \in X_{1}\right\}$ is a kTDS of $G_{I}$ with cardinal at most $\left|S_{F} \cap V\left(G_{I}\right)\right|+k$. If either $\left|S_{F} \cap X_{1}\right|=k$ and $\operatorname{deg}_{G}\left(x_{1}\right)=k+1$ or $\left|S_{F} \cap X_{1}\right|=k-1$ and $\operatorname{deg}_{G}\left(x_{1}\right)=k$, then for each $x_{1} x_{j} \in X_{1}-S_{F}$ the set $\left(S_{F} \cap V\left(G_{I}\right)\right) \cup\left\{x_{1} x_{j}, x_{j} x_{1}\right\}$ is a kTDS of $G_{I}$. Finally, if either $\left|S_{F} \cap X_{1}\right|=k$ and $\operatorname{deg}_{G}\left(x_{1}\right) \geq k+2$ or $\left|S_{F} \cap X_{1}\right|=k-1$ and $\operatorname{deg}_{G}\left(x_{1}\right) \geq k+1$, then for every two disjoint vertices $x_{1} x_{j}, x_{1} x_{i} \in X_{1}-S_{F}$, the set $\left(S_{F} \cap V\left(G_{I}\right)\right) \cup\left\{x_{1} x_{j}, x_{1} x_{i}\right\}$ is a kTDS of $G_{I}$. Thus in the Case 2 we proved that $\gamma_{\times k, t}\left(G_{I}\right)+\gamma_{\times k, t}\left(H_{I}\right)-k \leq \gamma_{\times k, t}\left(F_{I}\right)$.

With comparing the obtained bounds in Cases 1 and 2 , we have $\gamma_{\times k, t}\left(G_{I}\right)+\gamma_{\times k, t}\left(H_{I}\right)-k \leq$ $\gamma_{\times k, t}\left(F_{I}\right)$, and this completes our proof.

By closer look at the proof of Theorem 9 we have the next theorem.

Theorem 10. Let $F$ be a graph with a cut-edge e such that $G$ and $H$ are the components of $F-e$. If $2 \leq k<\min \{\delta(G), \delta(H)\}$, then

$$
\gamma_{\times k, t}\left(G_{I}\right)+\gamma_{\times k, t}\left(H_{I}\right)-2 \leq \gamma_{\times k, t}\left(F_{I}\right) \leq \gamma_{\times k, t}\left(G_{I}\right)+\gamma_{\times k, t}\left(H_{I}\right) .
$$

We now calculate the $k$-tuple total domination number of the inflation of the complete graphs and then continue our discussion.

Proposition 11. Let $n>k \geq 2$. Then every complete graph $K_{n}$ is $\lfloor(n-1) / 2\rfloor$-Hamiltonian-like decomposable graph and

$$
\gamma_{\times k, t}\left(\left(K_{n}\right)_{I}\right)=\left\{\begin{array}{cc}
n k+1 & \text { if } k \text { and } n \text { are both odd } \\
n k & \text { otherwise }
\end{array} .\right.
$$

Proof. Let $V(G)=\{i \mid 1 \leq i \leq n\}$. Since for any $1 \leq i \leq\lfloor(n-1) / 2\rfloor$ the edge set $E_{i}=\{(j, j+i) \mid$ $1 \leq j \leq n\}$ is a union of some disjoint cycles and $\cup_{1 \leq i \leq\lfloor(n-1) / 2\rfloor} E_{i}$ is a partition of $V\left(K_{n}\right)$, then $K_{n}$ is $\lfloor(n-1) / 2\rfloor$-Hamiltonian-like decomposable graph. Since $M=\{(i, i+\lfloor n / 2\rfloor) \mid 1 \leq i \leq\lfloor n / 2\rfloor\}$ is respectively a perfect or maximum matching of size $\lfloor n / 2\rfloor$ of $K_{n}$, when $n$ is respectively even or odd, then Theorems 5 and 8 complete our proof.

Proposition 12. Let $2 \leq k<n \leq m$ and let $F$ be a graph with a cut-edge e such that $G=K_{n}$ and $H=K_{m}$ are the components of $F-e$. Then

$$
\gamma_{\times k, t}\left(F_{I}\right)=\left\{\begin{array}{c}
k(n+m)+1 \\
k(n+m)
\end{array} \quad \text { if } k \text { is odd and } n \equiv m+1(\bmod 2) .\right.
$$

Proof. Let $V(G)=\left\{x_{i} \mid 1 \leq i \leq n\right\}, V(H)=\left\{y_{i} \mid 1 \leq i \leq m\right\}$ and $e=x_{n} y_{m}$. Since every complete graph $K_{t}$ is $\lfloor(t-1) / 2\rfloor$-Hamiltonian-like decomposable graph and $n \leq m$, then $F$ is $\lfloor(n-1) / 2\rfloor$ Hamiltonian-like decomposable graph. We now continue our discussion in the next two cases.

Case 1. $n \equiv m+1(\bmod 2)$.

If $k$ is odd, then Theorem [6 follows that $\gamma_{\times k, t}\left(F_{I}\right) \geq k(n+m)+1$. Without loss of generality, we may assume that $n$ is odd and $m$ is even. Then $\gamma_{\times k, t}\left(G_{I}\right)=k n+1$ and $\gamma_{\times k, t}\left(H_{I}\right)=k m$, by Proposition [1]. If $S_{G}$ and $S_{H}$ are respectively $\gamma_{\times k, t}\left(G_{I}\right)$-set and $\gamma_{\times k, t}\left(H_{I}\right)$-set, then $S_{G} \cup S_{H}$ is a kTDS of $F_{I}$ with cardinal $k(n+m)+1$ and so $\gamma_{\times k, t}\left(F_{I}\right)=k(n+m)+1$. If $k$ is even, then similarly it can be verified that $\gamma_{\times k, t}\left(F_{I}\right)=k(n+m)$.

Case 2. $n \equiv m(\bmod 2)$.

Then Theorem 1 follows that $\gamma_{\times k, t}\left(F_{I}\right) \geq k(n+m)$. If either $n \equiv m \equiv 0(\bmod 2)$ or $n \equiv m \equiv$ $1(\bmod 2)$ and $k$ is even, then $\gamma_{\times k, t}\left(G_{I}\right)=k n$, and $\gamma_{\times k, t}\left(H_{I}\right)=k m$, by Proposition [1] If $S_{G}$ and $S_{H}$ are respectively $\gamma_{\times k, t}\left(G_{I}\right)$-set and $\gamma_{\times k, t}\left(H_{I}\right)$-set, then obviously $S_{G} \cup S_{H}$ is a kTDS of $F_{I}$ with cardinal $k(n+m)$ and so $\gamma_{\times k, t}\left(F_{I}\right)=k(n+m)$.

Let now $n \equiv m \equiv 1(\bmod 2)$ and let $k$ be odd. Then $\gamma_{\times k, t}\left(G_{I}\right)=k n+1$ and $\gamma_{\times k, t}\left(H_{I}\right)=k m+1$, by Proposition 11, Let $S_{G}=S_{1} \cup\{\alpha, \beta\}$ be the given $\gamma_{\times k, t}\left(G_{I}\right)$-set in the second paragraph of the 
proof of Theorem 8 such that $S_{1}=V\left(M_{I}\right) \cup S^{(1)} \cup S^{(2)} \cup \ldots \cup S^{(k)}$ and $\alpha, \beta \in X_{n}-\left(S_{1}-V\left(M_{I}\right)\right)$. Similarly, let $S_{H}=S_{1}^{\prime} \cup\left\{\alpha^{\prime}, \beta^{\prime}\right\}$ be the given $\gamma_{\times k, t}\left(H_{I}\right)$-set in the second paragraph of the proof of Theorem 8 such that $S_{1}^{\prime}=V\left(M_{I}^{\prime}\right) \cup S^{\prime(1)} \cup S^{\prime(2)} \cup \ldots \cup S^{\prime(k)}$ and $\alpha^{\prime}, \beta^{\prime} \in Y_{m}-\left(S_{1}^{\prime}-V\left(M_{I}^{\prime}\right)\right)$. Then obviously $S=\left(\left(S_{G} \cup S_{H}\right)-\left\{\alpha, \beta, \alpha^{\prime}, \beta^{\prime}\right\}\right) \cup\left\{x_{n} y_{m}, y_{m} x_{n}\right\}$ is a kTDS of $F_{I}$ with cardinal $k(n+m)$ and so $\gamma_{\times k, t}\left(F_{I}\right)=k(n+m)$.

Proposition 11 follows that if $G=K_{n}$ and $H=K_{m}$ are complete graphs, then

$$
\gamma_{\times k, t}\left(G_{I}\right)+\gamma_{\times k, t}\left(H_{I}\right)= \begin{cases}k(n+m) & \text { if } k \text { is odd and } m \text { and } n \text { are both even, } \\ k(n+m)+1 & \text { if } k \text { is odd and } n \equiv m+1(\bmod 2), \\ k(n+m)+2 & \text { if } k, m \text { and } n \text { are odd }\end{cases}
$$

Thus Proposition 12 follows the next result that states the given bounds in Theorem 9 are sharp.

Corollary 13. Let $2 \leq k<n \leq m$ and let $F$ be a graph with a cut-edge e such that $G=K_{n}$ and $H=K_{m}$ are the components of $F-e$. Then

$$
\gamma_{\times k, t}\left(F_{I}\right)=\left\{\begin{array}{cc}
\gamma_{\times k, t}\left(G_{I}\right)+\gamma_{\times k, t}\left(H_{I}\right)-2 & \text { if } k, m \text { and } n \text { are odd } \\
\gamma_{\times k, t}\left(G_{I}\right)+\gamma_{\times k, t}\left(H_{I}\right) & \text { otherwise }
\end{array}\right.
$$

Now in the next theorem we give upper and lower bounds for the $k$-tuple total domination number of the inflation of a graph $F$, which contains a cut-vertex $v$, in terms on the $k$-tuple total domination numbers of the inflation of the $v$-components of $F-v$.

Theorem 14. Let $F$ be a graph with a cut-vertex $v$ such that $G^{1}, G^{2}, \ldots, G^{m}$ are all v-components of $F-v$. If $2 \leq k<\min \left\{\delta\left(G^{i}\right) \mid 1 \leq i \leq m\right\}$, then

$$
\Sigma_{1 \leq i \leq m} \gamma_{\times k, t}\left(G_{I}^{i}\right)-m(k+1)+k \leq \gamma_{\times k, t}\left(F_{I}\right) \leq \Sigma_{1 \leq i \leq m} \gamma_{\times k, t}\left(G_{I}^{i}\right),
$$

and the upper bound $\Sigma_{1 \leq i \leq m} \gamma_{\times k, t}\left(G_{I}^{i}\right)$ is sharp.

Proof. Let $V\left(G^{i}\right)=\left\{x_{j}^{i} \mid 1 \leq j \leq n\left(G^{i}\right)\right\}$. Without loss of generality, we may suppose that $x_{1}^{1}=\ldots=x_{1}^{m}=v$. Then $V\left(F_{I}\right)=\cup_{1 \leq i \leq m} V\left(G_{I}^{i}\right)$ and

$$
E\left(F_{I}\right)=\left(\cup_{1 \leq i \leq m} E\left(G_{I}^{i}\right)\right) \cup\left\{\left(x_{1}^{i} x_{l}^{i}, x_{1}^{j} x_{t}^{j}\right) \mid x_{1}^{i} x_{l}^{i} \in X^{i} \text {, and } x_{1}^{j} x_{t}^{j} \in X^{j} \text {, for } 1 \leq i<j \leq m\right\} .
$$

Let $S^{i}$ be a $\gamma_{\times k, t}\left(G_{I}^{i}\right)$-set. Since $\cup_{1 \leq i \leq m} S^{i}$ is a kTDS of $F_{I}$ with cardinal $\Sigma_{1 \leq i \leq m} \gamma_{\times k, t}\left(G_{I}^{i}\right)$, then $\gamma_{\times k, t}\left(F_{I}\right) \leq \Sigma_{1 \leq i \leq m} \gamma_{\times k, t}\left(G_{I}^{i}\right)$

Let now $S$ be a $\gamma_{\times k, t}\left(F_{I}\right)$-set. Let $S^{i}=S \cap V\left(G_{I}^{i}\right)$, where $1 \leq i \leq m$. Then each $S^{i}$ is a kTDS of $G_{I}^{i}-X_{1}^{i}$. Let $\left|S \cap X_{1}^{i}\right|=t_{i}$, where $1 \leq i \leq m$. Then $\Sigma_{1 \leq i \leq m} t_{i} \geq k$. Since $k<\delta\left(G^{i}\right)$, then by adding at most $k+1-t_{i}$ vertices of $X_{1}^{i}$ to $S$, we may obtain a kTDS $S^{\prime}$ of $F_{I}$ such that every $S^{\prime} \cap V\left(G_{I}^{i}\right)$ is a kTDS of $G_{I}^{i}$. Then

$$
\begin{aligned}
\Sigma_{1 \leq i \leq m} \gamma_{\times k, t}\left(G_{I}^{i}\right) & \leq \Sigma_{1 \leq i \leq m}\left|S_{F}^{\prime} \cap V\left(G_{I}^{i}\right)\right| \\
& \leq\left|S_{F}\right|+m(k+1)-\Sigma_{1 \leq i \leq m} t_{i} \\
& \leq \gamma_{\times k, t}\left(F_{I}\right)+m(k+1)-k
\end{aligned}
$$

and hence

$$
\Sigma_{1 \leq i \leq m} \gamma_{\times k, t}\left(G_{I}^{i}\right)-m(k+1)+k \leq \gamma_{\times k, t}\left(F_{I}\right) \leq \Sigma_{1 \leq i \leq m} \gamma_{\times k, t}\left(G_{I}^{i}\right) .
$$

Now we show that the upper bound $\Sigma_{1 \leq i \leq m} \gamma_{\times k, t}\left(G_{I}^{i}\right)$ is sharp. Let $F$ be a graph with a cutvertex $v$ such that $G^{1}, G^{2}, \ldots, G^{m}$ are all $v$-components of $F-v$ and $\gamma_{\times k, t}\left(G_{I}^{i}\right)=n\left(G^{i}\right) k$. Consider $V\left(G^{i}\right)=\left\{x_{j}^{i} \mid 1 \leq j \leq n\left(G^{i}\right)\right\}$ and $x_{1}^{1}=\ldots=x_{1}^{m}=v$. Let $Y_{v}^{F}$ be the respective red clique with vertex $v$ in $F$. Let $S^{i}$ be a $\gamma_{\times k, t}\left(G_{I}^{i}\right)$-set, where $1 \leq i \leq m$. Then every clique in $G_{I}^{i}$ contains exactly $k$ vertices of $S^{i}$. Then $S=\cup_{1 \leq i \leq m} S^{i}$ is a kTDS of $F_{I}$ with cardinal $\Sigma_{1 \leq i \leq m} \gamma_{\times k, t}\left(G_{I}^{i}\right)=$ $\Sigma_{1 \leq i \leq m} n\left(G^{i}\right) k$ such that $Y_{v}^{F}$ contains $m k$ vertices of $S$.

We claim that $S$ has minimum cardinal among of all $k$-tuple total dominating sets of $F_{I}$. Observation 7 follows that every red clique other than $Y_{v}^{F}$ must contain at least $k$ vertices of every kTDS of $F_{I}$. Thus we can not reduce the number of vertices of $S$ in cliques except probably $Y_{v}^{F}$. Since also reducing the number of the vertices of $S \cap Y_{v}^{F}$ reduce the cardinal of $k$-tuple total domination number of $G_{I}^{i}$, then we can not reduce it, by Observation 7 . Therefore $S$ is a minimal kTDS of 
$F_{I}$. Now let $S^{\prime}$ be an arbitrary $\gamma_{\times k, t}\left(F_{I}\right)$-set with cardinal less than $\Sigma_{1 \leq i \leq m} n\left(G^{i}\right) k$. Then, by the previous discussion, there exists a $v$-component $G^{i}$ of $F-v$ and a clique $X$ of it other than $X_{1}^{i}=Y_{v}^{F} \cap V\left(G_{I}^{i}\right)$ such that $\left|S^{\prime} \cap X\right|<k$. But this is not possible, by Observation 7 Therefore $S$ is a $\gamma_{\times k, t}\left(F_{I}\right)$-set and so $\gamma_{\times k, t}\left(F_{I}\right)=\Sigma_{1 \leq i \leq m} \gamma_{\times k, t}\left(G_{I}^{i}\right)=\Sigma_{1 \leq i \leq m} n\left(G^{i}\right) k$.

Let $G^{1}, G^{2}, \ldots, G^{m}$ and $F$ be the given graphs in the second part of the proof of Theorem 14. Then we see that $n(F)=\Sigma_{1 \leq i \leq m} n\left(G^{i}\right)-m+1$ and

$$
\begin{aligned}
\gamma_{\times k, t}\left(F_{I}\right) & =\Sigma_{1 \leq i \leq m} n\left(G^{i}\right) k \\
& =n(F) k+(m-1) k \\
& \leq n(F)(k+1)-1 .
\end{aligned}
$$

Thus this family of graphs are examples of the graphs $G$ of order $n$, which $\gamma_{\times k, t}\left(G_{I}\right)=n k+\alpha k \leq$ $n(k+1)-1$, where $\alpha$ is an arbitrary positive integer.

\section{4. $k$-TUPLE TOTAL DOMINATION NUMBER IN THE INFLATION OF SOME GRAPHS}

In section 3 , we calculated the $k$-tuple total domination number of the inflation of the complete graphs. Now we find this number in the inflation of the generalized Petersen graphs, Harary graphs and complete bipartite graphs. Also we give an upper bound for this number when our graph is a complete multipartite graph.

In 10, Watkins introduced the notion of generalized Petersen graph (GPG for short) as follows: for any integer $n \geq 3$ let $Z_{n}$ be additive group on $\{1,2, \ldots, n\}$ and $m \in Z_{n}-\{0\}$, the graph $P(n, m)$ is defined on the set $\left\{a_{i}, b_{i} \mid i \in Z_{n}\right\}$ of $2 n$ vertices with edges $a_{i} a_{i+1}, a_{i} b_{i}, b_{i} b_{i+m}$ for all $i$. If $m=n / 2$, then every vertex $b_{i}$ has degree 2 and every vertex $a_{i}$ has degree 3 , and otherwise $P(n, m)$ is 3-regular. Thus $\gamma_{\times 3, t}\left(\left(P(n, m)_{I}\right)=n\left(G_{I}\right)=6 n\right.$, where $m \neq n / 2$. Since $M=\left\{a_{i} b_{i} \mid i \in Z_{n}\right\}$ is a perfect matching in $P(n, m)$, then $S=\left\{a_{i} b_{i}, b_{i} a_{i} \mid i \in Z_{n}\right\}$ is a $\gamma_{t}\left((P(n, m))_{I}\right)$-set and so $\gamma_{t}\left(\left(P(n, m)_{I}\right)=2 n\right.$. In the next proposition we calculate $\gamma_{\times 2, t}\left(\left(P(n, m)_{I}\right)\right.$.

Proposition 15. Let $n \geq 3$ and $m \geq 1$ be integers. Then

$$
\gamma_{\times 2, t}\left((P(n, m))_{I}\right)=\left\{\begin{array}{cc}
4 n+2 & \text { if } m=n / 2 \text { is odd } \\
4 n & \text { otherwise }
\end{array} .\right.
$$

Proof. Let $G=P(n, m)$. We first assume that $m \neq n / 2$ and $d$ is the greatest common divisor of $m$ and $n$. Then the induced subgraph by $\left\{b_{i} \mid i \in Z_{n}\right\}$ of $G$ has a partition to $d$ disjoint cycle or cycles $C_{i}: b_{i} b_{i+m} b_{i+2 m} \ldots b_{i+\alpha-m}$, where $1 \leq i \leq d$ and $\alpha=\min \{t m \mid t m \equiv 0 \bmod n\}$. Since the induced subgraph by $\left\{a_{i} \mid i \in Z_{n}\right\}$ of $G$ is cycle $C_{a}: a_{1} a_{2} a_{3} \ldots a_{n}$, then $G$ is a Hamiltonian-like decomposable graph and Theorem 4 follows $\gamma_{\times 2, t}\left(G_{I}\right)=4 n$.

Let now $m=n / 2$. Then $b_{i} b_{j} \in E(G)$ if and only if $j \equiv i+m(\bmod n)$. Then every vertex $b_{i}$ has degree 2 and every vertex $a_{i}$ has degree 3 . Then there exist $\lfloor m / 2\rfloor$ disjoint cycles $b_{i} a_{i} a_{i+1} b_{i+1} b_{i+1+m} a_{i+1+m} a_{i+m} b_{i+m}$ of length 8 . If $m$ is even, then these cycles are a partition of $V(G)$. Hence $G$ is a Hamiltonian-like decomposable graph and Theorem 4 follows $\gamma_{\times 2, t}\left(G_{I}\right)=4 n$. Otherwise these cycles are a partition of $V(G)-\left\{a_{m}, b_{m}, b_{n}, a_{n}\right\}$. We notice that the induced subgraph of $G$ by $\left\{a_{m}, b_{m}, b_{n}, a_{n}\right\}$ is the path $P_{4}: a_{m} b_{m} b_{n} a_{n}$. Set

$$
\begin{aligned}
S & =S_{1} \cup S_{2} \cup \ldots \cup S_{m^{\prime}} \\
& \cup\left\{a_{m} a_{m+1}, a_{m} a_{m-1}, a_{m} a_{m} ; b_{m} a_{m}, b_{m} b_{n} ; b_{n} b_{m}, b_{n} a_{n} ; a_{n} b_{n}, a_{n} a_{1}, a_{n} a_{n-1}\right\},
\end{aligned}
$$

where $1 \leq i \leq m^{\prime}$ and

$$
\begin{aligned}
S_{i} & =\left\{b_{i} b_{i+m}, b_{i} a_{i} ; a_{i} b_{i}, a_{i} a_{i+1} ; a_{i+1} a_{i}, a_{i+1} b_{i+1} ; b_{i+1} a_{i+1},\right\} \\
& \cup\left\{b_{i+1} b_{i+1+m} ; b_{i+1+m} b_{i+1}, b_{i+m+1} a_{i+m+1} ; a_{i+m+1} b_{i+m+1}\right\} \\
& \cup\left\{a_{i+m+1} a_{i+m} ; a_{i+m} a_{i+m+1}, a_{i+m} b_{i+m} ; b_{i+m} a_{i+m}, b_{i+m} b_{i}\right\} .
\end{aligned}
$$

One can verify that $S$ is a minimum DTDS of $G_{I}$ and so $\gamma_{\times 2, t}\left(G_{I}\right)=4 n+2$.

We now consider Harary graphs which make a great family of graphs. Given $m<n$, place $n$ vertices $1,2, \ldots, n$ around a circle, equally spaced. If $m$ is even, form $H_{m, n}$ by making each vertex adjacent to the nearest $m / 2$ vertices in each direction around the circle. If $m$ is odd and $n$ is even, form $H_{m, n}$ by making each vertex adjacent to the nearest $(m-1) / 2$ vertices in each direction and 
to the diametrically opposite vertex. In each case, $H_{m, n}$ is $m$-regular. When $m$ and $n$ are both odd, index the vertices by the integers modulo $n$. Construct $H_{m, n}$ from $H_{m-1, n}$ by adding the edges $(i, i+(n-1) / 2)$, for $0 \leq i \leq(n-1) / 2$ (see [11]).

Proposition 16. Let $2 \leq k \leq m<n$ be integers. Then the Harary graph $H_{m, n}$ is $\lfloor m / 2\rfloor$ Hamiltonian-like decomposable graph and

$$
\gamma_{\times k, t}\left(\left(H_{m, n}\right)_{I}\right)=\left\{\begin{array}{cc}
n k+1 & \text { if } k \text { and } n \text { are both odd } \\
n k & \text { otherwise }
\end{array} .\right.
$$

Proof. Since for each $1 \leq i \leq m$ the edge subset $E_{i}=\{(j, j+i) \mid 1 \leq j \leq n\}$ is a union of some disjoint cycles and $\cup_{1 \leq i \leq m} E_{i}$ is a partition of $V\left(H_{m, n}\right)$, then $H_{m, n}$ is a $m$-Hamiltonian-like decomposable graph. Let $m$ be odd. If $n$ is even or odd, then $M=\{(i, i+\lfloor n / 2\rfloor) \mid 1 \leq i \leq\lfloor n / 2\rfloor\}$ is respectively a perfect or maximum matching of size $\lfloor n / 2\rfloor$ of $H_{m, n}$. Then Theorems 5 and 8 complete our proof.

In the following two theorems we consider the complete bipartite graphs $K_{p, q}$. First let $p=q$.

Proposition 17. For integers $p \geq k \geq 2$, let $G$ be the complete bipartite graph $K_{p, p}$. Then $G$ is a $(\lfloor p / 2\rfloor-1) H L P M$-graph if $p$ is even, otherwise is a $\lfloor p / 2\rfloor$-Hamiltonian-like decomposable graph and so $\gamma_{\times k, t}\left(G_{I}\right)=2 p k$.

Proof. We consider the partition $X \cup Y$ for $V(G)$, where $X=\left\{x_{i} \mid 1 \leq i \leq p\right\}$ and $Y=\left\{y_{i} \mid 1 \leq i \leq\right.$ $p\}$. For $0 \leq j \leq\lfloor p / 2\rfloor-1$, we choose $\lfloor p / 2\rfloor$ sequences on $X \cup Y$ of length $2 p$ that are alternatively from $X$ and $Y$ with starting of vertex $x_{1}$ such that every three consequence numbers of them are $x_{i}, y_{i+j}$, and $x_{i+(2 j+1)}$. Let $0 \leq j \leq\lfloor p / 2\rfloor-2$. If $p$ does not divided by $2 j+1$, then $j$-th sequence makes the cycle

$$
C_{j}: x_{1} y_{j+1} x_{2 j+2} y_{3 j+2} \ldots x_{p-2 j} y_{p-j}
$$

but if $p=(2 j+1) t$, for some positive integer $t$, then it makes $2 j+1$ disjoint cycles

$$
C_{i}^{j}: x_{i} y_{i+j} x_{i+(2 j+1)} y_{i+(3 j+1)} \ldots x_{i+(t-1)(2 j+1)} y_{i+(t-1)(2 j+1)+j}
$$

of length $t$, where $1 \leq i \leq 2 j+1$. We notice that for odd $p$ and $j=\lfloor p / 2\rfloor-1$ there exists another cycle of length $2 p$ that is disjoint of the other cycles. When $p$ is even and $j=\lfloor p / 2\rfloor-1$, the corresponding sequence makes a perfect matching $M$ that is disjoint of the other cycles. Then Theorems 4 and 5 follow $\gamma_{\times k, t}\left(G_{I}\right)=2 p k$.

Proposition 18. For integers $q \geq p>k \geq 2$, let $G$ be the complete bipartite graph $K_{p, q}$. Then $\gamma_{\times k, t}\left(G_{I}\right)=2 p k+(q-p)(k+1)$.

Proof. Let $S$ be an arbitrary $\gamma_{\times k, t}\left(G_{I}\right)$-set such that $\alpha$ red cliques of $G_{I}$ contain $k$ vertices and other $p+q-\alpha$ red cliques of $G_{I}$ contain $k+1$ vertices of $S$. Since $G$ is bipartite, then $\alpha / 2$ cliques must be selected among of the $q$ red cliques $Y_{i}$, where $1 \leq i \leq q$, and the other second $\alpha / 2$ cliques must be selected among of the $p$ red cliques $X_{i}$, where $1 \leq i \leq p$. We notice that this choosing is possible. Because, by Proposition 17, $K_{p, p}$ is respectively $(\lfloor p / 2\rfloor-1)$ HLPM-graph and $\lfloor p / 2\rfloor$-Hamiltonian-like decomposable graph, when $p$ is respectively even or odd. Thus $\alpha \leq 2 p$ and so

$$
\begin{aligned}
\gamma_{\times k, t}\left(G_{I}\right) & =\min \left\{|S|: S \text { is a kTDS of } G_{I}\right\} \\
& =\min \{\alpha k+(q+p-\alpha)(k+1): 0 \leq \alpha \leq 2 p\} \\
& =\min \{(q+p)(k+1)-\alpha: 0 \leq \alpha \leq 2 p\} \\
& =(q+p)(k+1)-2 p \\
& =2 p k+(q-p)(k+1) .
\end{aligned}
$$

We notice that $\gamma_{\times p, t}\left(\left(K_{p, q}\right)_{I}\right)=2 p q$ and for $k=n, 2 p q=2 p k+(q-p)(k+1)$ if and only if $p=q$. By Theorem 1 if $k \geq 2$ is integer and $G$ is a graph of order $n$ with $\delta \geq k$, then $n(k+1)-n \leq \gamma_{\times k, t}\left(G_{I}\right) \leq n(k+1)-1$. Therefore Proposition 18 follows the next theorem.

Theorem 19. For each integers $n, k$ and $\ell$ with the condition $2 \leq k<\ell \leq\lfloor n / 2\rfloor$, there exists a graph $G$ of order $n$ such that $\gamma_{\times k, t}\left(G_{I}\right)=n(k+1)-2 \ell$. 
Proof. Let $G=K_{\ell, n-\ell}$. Then Proposition 18 follows

$$
\begin{aligned}
\gamma_{\times k, t}\left(G_{I}\right) & =2 \ell k+(n-2 \ell)(k+1) \\
& =n(k+1)-2 \ell .
\end{aligned}
$$

The next theorem gives an upper bound for the $k$-tuple total domination number of the complete multipartite graphs.

Proposition 20. Let $G$ be the complete multipartite graph $K_{n_{1}, n_{2}, \ldots, n_{m}}$. Let $n=n_{1}+\ldots+n_{m}$ and $n^{\prime}=\max \left\{\sum_{i \in J} n_{i} \mid J \subseteq\{1,2, . . m\}\right.$ and $\left.\sum_{i \in J} n_{i} \leq n / 2\right\}$. Then for every $2 \leq k<n^{\prime}$,

$$
\gamma_{\times k, t}\left(G_{I}\right) \leq n(k+1)-2 n^{\prime} .
$$

Proof. We assume that $V(G)=X^{(1)} \cup X^{(2)} \cup \ldots \cup X^{(m)}$ is the partition of vertices of the graph, where $X^{(i)}=\left\{x_{j}^{(i)} \mid 1 \leq j \leq n_{i}\right\}$. Let $n^{\prime}=\sum_{i \in J} n_{i}$, for some $J \subseteq\{1,2, . . m\}$. Let $X=\cup_{i \in J} X^{(i)}$ and $Y=\cup_{i \notin J} X^{(i)}$. Then every vertex of $X$ is adjacent to every vertex of $Y$. If $H$ is the complete bipartite with the vertex set $X \cup Y$, then it is a subgraph of $G$ and so $\gamma_{\times k, t}\left(G_{I}\right) \leq \gamma_{\times k, t}\left(H_{I}\right)=n(k+1)-2 n^{\prime}$, by Proposition 18 .

In the end of our paper we expose some problems.

Problems:

1. Can be improved the upper bound $n(k+1)-1$ in Theorem 1?

2. Whether the lower bound $\Sigma_{1 \leq i \leq m} \gamma_{\times k, t}\left(G_{I}^{i}\right)-m(k+1)+k$ in Theorem 14 is sharp?

3. Characterize all graphs $G$ such that $\gamma_{\times k, t}\left(G_{I}\right)=n k+1$.

\section{REFERENCES}

[1] F. Harary and T. W. Haynes, Double domination in graphs, Ars Combin. 55 (2000) 201-213.

$[2]$ T. W. Haynes, S. T. Hedetniemi, and P. J. Slater (Eds.), Fundamentals Domination in Graphs, Marcel Dekker, Inc. New York, 1998.

[3] T. W. Haynes, S. T. Hedetniemi, and P. J. Slater (Eds.), Domination in Graphs: Advanced Topics, Marcel Dekker, Inc. New York, 1998.

[4] M. A. Henning, A. P. Kazemi, k-Tuple Total Domination Number in Graphs, Discrete Applied Mathematics, 158 (2010) 1006-1011.

[5] M. A. Henning, A. P. Kazemi, Total Domination in Inflated Graphs, Manuscript 2010.

[6] J. E. Dunbar, T.W. Haynes, Domination in Infated Graphs, Congr. Numer. 118 (1996) 143-154.

[7] O. Favaron, Irredundance in Infated Graphs, J. Graph Theory 28 (1998) 97-104.

[8] O. Favaron, Infated Graphs with Equal Independent Number and Upper Irredundance Number, Discrete Mathematics 236 (2001) 81-94.

[9] J. Puech, The Lower Irredundance and Domination Parameters are Equal for Inflated Trees, J. Combin. Math. Combin. Comput. 33 (2000) 117-127.

[10] M. E. Watkins, A Theorem on Tait Coloring with an Application to the Generalized Petersen Graphs, J. Combin. Theory 6 (1969) 152-164.

[11] B. B. West, Introduction to Graph Theorey, 2nd ed., Prentice Hall, USA, 2001. 